\title{
A Novel Prognostic Nomogram for Gallbladder Cancer after Surgical Resection: A Single-Center Experience
}

\author{
Zuyi Ma $\mathbb{D}^{1,2}$ Fengying Dong, ${ }^{3}$ Zhenchong Li, ${ }^{2}$ Zehao Zheng, ${ }^{1,2}$ Zixuan Zhou, ${ }^{2}$ \\ Hongkai Zhuang $\mathbb{D},{ }^{1,2}$ Chunsheng Liu, ${ }^{1,2}$ Bowen Huang, ${ }^{4}$ Shanzhou Huang $\left(\mathbb{D},{ }^{2}\right.$ \\ Yiping Zou $\mathbb{D},{ }^{1,2}$ LinLing Yang, ${ }^{5}$ Yuanfeng Gong $\mathbb{D},{ }^{2,6}$ Chuanzhao Zhang $\mathbb{D}{ }^{2}$ \\ and Baohua Hou $\mathbb{D i D}^{2}$ \\ ${ }^{1}$ Shantou University of Medical College, Shantou 515000, China \\ ${ }^{2}$ Department of General Surgery, Guangdong Provincial People's Hospital, School of Medicine, \\ South China University of Technology, Guangzhou 510080, China \\ ${ }^{3}$ Forth Department of Geriatrics, General Hospital of Southern Theater Command, PLA, Guangzhou 510080, China \\ ${ }^{4}$ Department of General Surgery, Peking Union Medical College Hospital, \\ Chinese Academy of Medical Sciences and Peking Union Medical College, Beijing 100730, China \\ ${ }^{5}$ Guangzhou Medical University, Guangzhou 511436, China \\ ${ }^{6}$ Department of Hepatobiliary Surgery, The Affiliated Cancer Hospital and Institute of Guangzhou Medical University, \\ Guangzhou 510095, China
}

Correspondence should be addressed to Yuanfeng Gong; gongyuanfeng@gzhmu.edu.cn, Chuanzhao Zhang; 641703837@qq.com, and Baohua Hou; hbh1000@126.com

Received 7 December 2020; Revised 16 January 2021; Accepted 23 January 2021; Published 8 February 2021

Academic Editor: Bipul Acharya

Copyright ( 2021 Zuyi Ma et al. This is an open access article distributed under the Creative Commons Attribution License, which permits unrestricted use, distribution, and reproduction in any medium, provided the original work is properly cited.

\begin{abstract}
Background. Gallbladder cancer (GBC), which accounts for more than $80 \%$ of biliary tract malignancies, has a poor prognosis with an overall 5-year survival less than $10 \%$. The study aimed to identify risk factors and develop a predictive model for GBC following surgical resection. Methods. 98 GBC patients who underwent surgical resection from Guangdong Provincial People's Hospital were enrolled in the study. Cox-regression analysis was performed to identify significant prognostic factors. A nomogram was constructed and Harrell's concordance index, calibration plot, and decision cure analysis were used to evaluate the discrimination and calibration of the nomogram. Results. Liver resection, tumor size, perineural invasion, surgical margin, and liver invasion were identified as independent risk factors for overall survival (OS) in GBC patients who underwent surgical resection. Based on the selected risk factors, a novel nomogram was constructed. The C-index of the nomogram was 0.777, which was higher than the American Joint Committee on Cancer (AJCC) staging system (0.724) and Nevin staging system (0.659). Decision cure analysis revealed that the nomogram had a better net benefit and the calibration curves for the 1-, 3-, and 5-year survival probabilities were also well matched with the actual survival rates. Lastly, high-risk GBC were stratified based on the scores of the nomogram and we found high-risk GBC were associated with both worse OS and disease-free survival (DFS). Conclusion. We developed a nomogram showing a better predictive capacity for patients' survival of resected GBC than the AJCC staging systems. The established model may help to stratify high-risk GBC and facilitate decision-making in the clinic.
\end{abstract}

\section{Introduction}

Gallbladder cancer (GBC) is relatively uncommon with an overall incidence of 2.3 per 100,000 people, but it accounts for more than $80 \%$ of biliary tract malignancies [1]. Patients with GBC have a poor prognosis with an overall 5-year survival less than $10 \%$ [2]. Some studies have suggested that elevated carbohydrate antigen 19-9 (CA19-9) and carcinoembryonic antigen (CEA) were associated with the poor prognosis of GBC $[3,4]$. Other clinicopathological characters including positive surgical margin, lympho-vascular invasion, perineural invasion, and liver invasion were also 
considered as the risk factors for GBC patients prognosis [5-7]. Nevin staging system was simple and convenient for surgeons to evaluate the GBC patients and approximately predicted the GBC prognosis [8]. Regarding traditional tumor stages, tumor-node-metastasis (TNM) staging from the American Joint Committee on Cancer (AJCC) has been considered as the most valuable predictor of GBC prognosis [9]. However, the staging systems mainly focus on pathological outcomes but ignore some demographic characteristics.

Recently, investigators tried to develop novel prognostic models for patients with $\mathrm{GBC}$ based on different cohorts $[10,11]$. Wang et al. established a nomogram to make individualized estimates of survival by using the Surveillance, Epidemiology, and End Results (SEER) Medicare database in 2011 [11]. They predicted that GBC patients with at least T2 or N1 disease would gain a survival benefit from adjuvant chemoradiotherapy. However, due to lack of much clinical information and various pathological factors, using SEER Medicare database for prediction model establishment could cause much bias.

In the current study, we identified prognostic factors from comprehensive tumor characteristics and surgical parameters based on the data of our institution. Furthermore, we developed a novel nomogram to predict the prognosis of patients with GBC after surgical resection.

\section{Methods}

2.1. Data Collection. From January 1st, 2008, to January 1, 2019 , data of patients who underwent cholecystectomy with or without partial hepatectomy and with pathologically confirmed GBC at Guangdong Provincial People's Hospital were reviewed and included in the present study. The inclusion criteria were as follows: (1) GBC was primarily diagnosed; (2) had undergone cholecystectomy with or without wedge liver resection and with pathologically confirmed GBC; (3) American Society of Anesthesiologists (ASA) Score < III; (4) no neoadjuvant treatments before surgery. Patients who (1) had incomplete clinical and pathological data, (2) had other malignancy, (3) had distant metastasis, and (4) died within 30 days after surgery were excluded from this study. Patients' demographic, clinical, and pathological information and corresponding variables were manually collected, including age, sex, body mass index (BMI), diabetes mellitus (DM), jaundice, gallbladder stone, serum albumin, CA19-9, carbohydrate antigen 125 (CA125), CEA, tumor size, surgical margin, liver resection, lymph node resection, tumor grade, lymphovascular invasion, perineural invasion, liver invasion, and lymph node positive rate (LNR). GBC stage and postoperative pathologic TNM (pTNM) information was determined by using the AJCC 8th edition (AJCC-8) classification system [9].

Different surgeries were performed for patients based on the TNM stage in our center: for patients at Tis or T1a stage, simple cholecystectomy was performed; for patients from T1b to T3 stage, not only cholecystectomy but also the wedge resection and lymph node dissection were performed to obtain the radical resection; for T4 stage, extended resection including peripheral organ resection was performed for getting a better prognosis. The primary end points were overall survival (OS) and disease-free survival (DFS), which were defined as the date of surgery to the death and recurrence or last follow-up, respectively. This study was approved by the Institutional Ethics Committee of the Guangdong Provincial People's Hospital.

\subsection{Statistical Analysis and Nomogram Construction.} Statistical analyses were performed by SPSS 20.0 and $R$ version 4.0.0 software (http://www.r-project.org/). $P$-value $<0.05$ was regarded as statistically significant and all tests were two-sided. Cutoff values for tumor size, CA19-9, CA125, CEA were all defined by the receiver operating characteristic curve (ROC) analyses with the help of SPSS 20.0. Cox proportional hazards regression was used to evaluate potential risk factors of prognosis. With the help of rms package of $R$, the visual nomogram based on the potential risk factors was constructed. The discrimination performance of the nomogram was assessed by using the concordance index (C-index). Overfit bias was decreased by bootstrap validation including 1,000 resamples. The calibration of the nomogram was estimated by using a calibration plot. Decision curve analysis (DCA) was conducted to assess the clinical performance and the net benefit of the nomogram. Patients were divided into a high-risk group and a low-risk group using the median cutoff of the risk score. Then, the log-rank tests and Kaplan-Meier analyses were performed using the survival $R$ package between the highrisk and low-risk group to assess the predictive ability of the prognostic nomogram.

\section{Results}

3.1. Patients Characteristics and Follow-Up. Based on the criteria, 98 eligible patients were finally enrolled into our study. Patients' clinicopathologic characteristics above are listed in Table 1. Based on the best cutoff values, tumor size, carbohydrate antigen 19-9, carbohydrate antigen 12-5, and carcinoembryonic antigen were divided into two groups and were set as cCA-19-9, cCA12-5, cCEA, and ctumor size. The median follow-up time was 28.5 months (range, 12-126 months). Overall, the median OS and DFS were 20 months and 14.5 months. The 1-, 3-, and 5-year OS and DFS rates were $73.3 \%, 48.8 \%$, and $19.2 \%$ and $65.5 \%, 46.83 \%$, and $14.7 \%$, respectively.

\subsection{Identification of Independent Prognostic Factors for GBC.} Cox regression analysis was performed to identify the prognostic factors for GBC. After performing the univariate cox regression analysis, jaundice, tumor grade, lymphovascular invasion, perineural invasion, surgical margin, liver invasion, cCA-19-9, cCA12-5, cCEA, and ctumor size were regarded as potential risk factors. All significant univariable predictors and some other important clinical variables (e.g., liver resection) were enrolled into multivariate cox regression analysis (Table 2). Finally, the results indicated that perineural invasion, surgical margin, liver invasion, liver 
TABLE 1: Clinicopathological characteristics of patients.

\begin{tabular}{|c|c|c|}
\hline \multicolumn{2}{|l|}{ Patient characteristics } & $\begin{array}{c}\text { Patient } \\
\text { number }(n=98)\end{array}$ \\
\hline \multirow{2}{*}{ Age (years) } & $\geq 60$ & 55 \\
\hline & $<60$ & 43 \\
\hline \multirow{2}{*}{ Gender } & Male & 42 \\
\hline & Female & 56 \\
\hline \multirow{2}{*}{ BMI $\left(\mathrm{kg} / \mathrm{m}^{2}\right)$} & $\geq 24$ & 20 \\
\hline & $<24$ & 78 \\
\hline \multirow{2}{*}{ Jaundice } & Yes & 20 \\
\hline & No & 78 \\
\hline \multirow{2}{*}{ Diabetes mellitus } & Yes & 15 \\
\hline & No & 83 \\
\hline \multirow{2}{*}{ Gallbladder stone } & Yes & 46 \\
\hline & No & 52 \\
\hline \multirow{4}{*}{ T stage } & Tis/T1 & 12 \\
\hline & $\mathrm{T} 2$ & 54 \\
\hline & $\mathrm{T} 3$ & 24 \\
\hline & $\mathrm{T} 4$ & 8 \\
\hline \multirow{2}{*}{ AJCC stage } & I IIIA & 67 \\
\hline & IIIB IVA & 31 \\
\hline \multirow{3}{*}{ Tumor grade } & Low & 29 \\
\hline & Medium & 61 \\
\hline & High & 13 \\
\hline \multirow{2}{*}{ Lymphovascular invasion } & Yes & 20 \\
\hline & No & 78 \\
\hline \multirow{2}{*}{ Perineural invasion } & Yes & 34 \\
\hline & No & 64 \\
\hline \multirow{2}{*}{ Surgical margin } & R0 & 80 \\
\hline & $\mathrm{R} 1$ & 18 \\
\hline \multirow{2}{*}{ Liver invasion } & Yes & 23 \\
\hline & No & 75 \\
\hline \multirow{2}{*}{ Lymph node positive rate (LNR) } & $\geq 0.28$ & 24 \\
\hline & $<0.28$ & 74 \\
\hline \multirow{2}{*}{ Liver resection } & Yes & 66 \\
\hline & No & 32 \\
\hline \multirow{2}{*}{ Lymph node dissection (LND) } & Yes & 55 \\
\hline & No & 43 \\
\hline \multirow{2}{*}{ Tumor size $(\mathrm{cm})$} & $\geq 4.5$ & 26 \\
\hline & $<4.5$ & 72 \\
\hline \multirow{2}{*}{ CA125 (U/ml) } & $\geq 12$ & 62 \\
\hline & $<12$ & 36 \\
\hline \multirow{2}{*}{ CA19-9 (U/ml) } & $\geq 58.5$ & 32 \\
\hline & $<58.5$ & 66 \\
\hline \multirow{2}{*}{ CEA (ng/ml) } & $\geq 5$ & 22 \\
\hline & $<5$ & 76 \\
\hline
\end{tabular}

BMI, body mass index; CA125, carbohydrate antigen 125; CA19-9, carbohydrate antigen 19-9; CEA, carcinoembryonic antigen; the American Joint Committee on Cancer (AJCC) stage is according to the AJCC 8th edition.

resection, cCA-19-9, and ctumor size were the independent prognostic factors for OS in patients with GBC who underwent the surgical therapy (Table 2). Further, we performed the Schoenfeld residuals analysis to evaluate whether the proportional hazards assumption was valid. We show in Figure 1 that the $P$-values for liver resection (Figure 1(a)), cCA19-9 (Figure 1(b)), ctumor size (Figure 1(c)), perineural invasion (Figure 1(d)), surgical margin (Figure 1(e)), and liver invasion (Figure 1(f)) are 0.4646, 0.0121, 0.9401, 0.6841, 0.8082 , and 0.7882 separately and the $P$-value for global test is 0.3456 . These results indicated that liver resection, ctumor size, perineural invasion, surgical margin, and liver invasion were satisfied for the proportional hazards assumption. However, cCA19-9 was unsatisfied and excluded in the multivariate model.

3.3. Construction and Validation of a Novel Prognostic Nomogram for OS. Based on the results of multivariate cox regression analysis, perineural invasion, surgical margin, liver invasion, liver resection, and ctumor size were selected as the significant factors and were integrated to establish a nomogram for predicting the OS of GBC (Figure 2). After applying the bootstrap validation, the bias-corrected $\mathrm{C}$-index of the nomogram was 0.777 , which was higher than the AJCC staging system (0.724) and Nevin staging system (0.659). In our OS nomogram, perineural invasion was given 100 points while R0 surgical margin was assigned 94.0578 points. The presence of liver invasion was assigned as 71.90517 points, while conducting the liver resection treatment would be given 44.78229 points. A tumor size less than $4.5 \mathrm{~cm}$ was scored as 0 , and a tumor size $\geq 4.5 \mathrm{~cm}$ was scored as 58.71448 points. Finally, the total points can be calculated and converted to obtain the probability of 1-, 3- and 5-year overall survival. The calibration curves for the 1-year (Figure 3(a)), 3-year (Figure 3(b)), and 5year (Figure 3(c)) survival probabilities were also well matched with the actual survival rates, which showed that the nomogram could predict the 1-, 3-, and 5-year OS in our cohort accurately. DCA were conducted to assess the clinical performance and the net benefit of the nomogram. It is revealed that the nomogram had a better net benefit with a wider range of threshold probabilities than AJCC staging system and Nevin stagging system for both 1-year (Figure 4(a)), 3-year (Figure 4(b)), and 5-year (Figure 4(c)) OS, which could provide a better clinical benefit for the clinicians and patients.

\subsection{The Association between High-Risk GBC and Patients'} Survival. The cohort was divided into high-risk group and low-risk group based on the median of the risk score and Kaplan-Meier analysis was performed to evaluate patients' OS and DFS in the two groups. The results showed that patients in high-risk group had both shorter OS $(P$-value $=3.768 e-07)$ and DFS $(P$-value $=2.359 e-06)$ than those in low-risk group (Figures 5(a) and 5(b)), indicating a significant unfavorable outcome for high-risk GBC.

\section{Discussion}

Despite continuous advances in the diagnosis and management of GBC in recent years, no major breakthrough for effective biomarkers nor treatment strategies have emerged. Surgical resection remains the only potentially curative treatment for patients with GBC [12]. However, the prognosis of GBC patient was still very poor with a 5-year survival rate of only about $10 \%$ [13]. For patients who underwent the surgical resection, it is of great interest to 
TABle 2: Univariate and multivariate Cox regression analyses for survival.

\begin{tabular}{|c|c|c|c|c|c|}
\hline \multirow{2}{*}{ Patient characteristics } & & \multicolumn{2}{|c|}{ Univariate analysis } & \multicolumn{2}{|c|}{ Multivariate analysis } \\
\hline & & HR $(95 \% \mathrm{CI})$ & $P$-value & HR (95\%CI) & $P$-value \\
\hline \multirow{2}{*}{ Age } & $<60$ & 1 & & & \\
\hline & $\geq 60$ & $1.484(0.826 \sim 2.666)$ & 0.187 & & \\
\hline \multirow{2}{*}{ Gender } & Male & 1 & & & \\
\hline & Female & $1.559(0.834 \sim 2.917)$ & 0.164 & & \\
\hline \multirow{2}{*}{ BMI } & $<24$ & 1 & & & \\
\hline & $\geq 24$ & $0.585(0.248 \sim 1.379)$ & 0.22 & & \\
\hline \multirow{2}{*}{ Jaundice } & Absent & 1 & & 1 & \\
\hline & Present & $2.182(1.105 \sim 4.308)$ & $<0.05$ & $1.407(0.624 \sim 3.175)$ & 0.411 \\
\hline \multirow{2}{*}{ Diabetes mellitus } & Absent & 1 & & & \\
\hline & Present & $0.917(0.362 \sim 2.319)$ & 0.855 & & \\
\hline \multirow{2}{*}{ Gallbladder stone } & Absent & 1 & & & \\
\hline & Present & $1.199(0.676 \sim 2.127)$ & 0.534 & & \\
\hline \multirow{2}{*}{ AJCC stage } & I IIIA & 1 & & & \\
\hline & IIIB-IVB & $3.66(1.963 \sim 6.825)$ & $<0.05$ & $1.269(0.422 \sim 3.811)$ & 0.671 \\
\hline \multirow{3}{*}{ Tumor grade } & High & 1 & & & \\
\hline & Medium & $7.049(0.952 \sim 52.170)$ & 0.056 & $5.707(0.731 \sim 44.527)$ & 0.10 \\
\hline & Low & $11.186(1.470 \sim 85.170)$ & 0.020 & $6.340(0.702 \sim 57.291)$ & 0.10 \\
\hline \multirow{2}{*}{ Lymphovascular invasion } & Absent & 1 & & 1 & \\
\hline & Present & $1.877(0.930 \sim 3.787)$ & 0.0788 & $1.4160(0.635 \sim 3.156)$ & 0.395 \\
\hline \multirow{2}{*}{ Perineural invasion } & Absent & 1 & & 1 & \\
\hline & Present & $3.949(2.189 \sim 7.122)$ & $<0.05$ & $3.411(1.671 \sim 6.963)$ & $<0.05$ \\
\hline \multirow{2}{*}{ Surgical margin } & R0 & 1 & & 1 & \\
\hline & $\mathrm{R} 1$ & 4.234(2.028 8.839) & $<0.05$ & $3.102(1.201 \sim 8.013)$ & $<0.05$ \\
\hline \multirow{2}{*}{ Liver invasion } & Absent & 1 & & 1 & \\
\hline & Present & $3.026(1.602 \sim 5.718)$ & $<0.05$ & $3.00(1.336 \sim 6.736)$ & $<0.05$ \\
\hline \multirow{2}{*}{ Lymph node positive rate } & $<0.28$ & 1 & & 1 & \\
\hline & $\geq 0.28$ & $3.007(1.502 \sim 6.020)$ & $<0.05$ & $1.019(0.319 \sim 3.251)$ & 0.975 \\
\hline \multirow{2}{*}{ Liver resection } & Absent & & & 1 & \\
\hline & Present & $0.771(0.426 \sim 1.395)$ & 0.39 & $0.3593(0.162 \sim 0.797)$ & $<0.05$ \\
\hline \multirow{2}{*}{ Tumor size } & $<4.5$ & 1 & & 1 & \\
\hline & $\geq 4.5$ & $1.807(0.9715 \sim 3.362)$ & 0.061 & $2.660(1.248 \sim 5.672)$ & $<0.05$ \\
\hline \multirow{2}{*}{ CA125 } & $<12$ & 1 & & 1 & \\
\hline & $\geq 12$ & $2.639(1.338 \sim 5.025)$ & $<0.05$ & $1.663(0.760 \sim 3.643)$ & 0.203 \\
\hline \multirow{2}{*}{ CA19-9 } & $<58.5$ & 1 & & 1 & \\
\hline & $\geq 58.5$ & $3.539(1.936 \sim 6.468)$ & $<0.05$ & $2.254(1.070 \sim 4.745)$ & $<0.05$ \\
\hline \multirow{2}{*}{ CEA } & $<5$ & 1 & & 1 & \\
\hline & $\geq 5$ & $3.155(1.622 \sim 6.136)$ & $<0.05$ & $0.558(0.237 \sim 1.314)$ & 0.182 \\
\hline
\end{tabular}

BMI, body mass index; CA125, carbohydrate antigen 125; CA19-9, carbohydrate antigen 19-9; CEA, carcinoembryonic antigen; the American Joint Committee on Cancer (AJCC) stage is according to the AJCC 8th edition.

develop an accurate prognostic model and identify the highrisk patients.

In the current study, perineural invasion, surgical margin, liver invasion, liver resection, and ctumor size were selected as the significant predictive factors and integrated to establish a nomogram for predicting the OS of GBC. The C-index of the nomogram was 0.775 , which was higher than the C-index for the 8th edition TNM stage system (0.724). The calibration plot revealed a good coincidence between predicted survival rate and actual survival rate and decision cure analysis also demonstrated that our nomogram had a strong potential clinical application. The Kaplan-Meier plot suggested that patients in high-risk group had significantly poorer recurrence-free survival and overall survival than those in low-risk group. The results above indicated that our nomogram had outstanding consistency, calibration, discrimination, and stratification for prediction of GBC patients who underwent surgical resection.

Perineural invasion is relatively common in GBC patients at T2 and T3 stage [14]. Some studies had reported that perineural invasion was significantly related to residual disease in incidental gallbladder carcinoma. Other researches also indicated that perineural invasion was associated with poor prognosis of GBC patients after surgical resection $[15,16]$. Perineural invasion was almost not observed in patients with stage $\mathrm{T} 1$ cancer, while it was detected rarely in distal-type tumors, which would develop various types of recurrences [15]. A current study indicated that an increasing affinity for nerve of tumor cells may be caused by a reciprocal interaction between the cancer cells and 


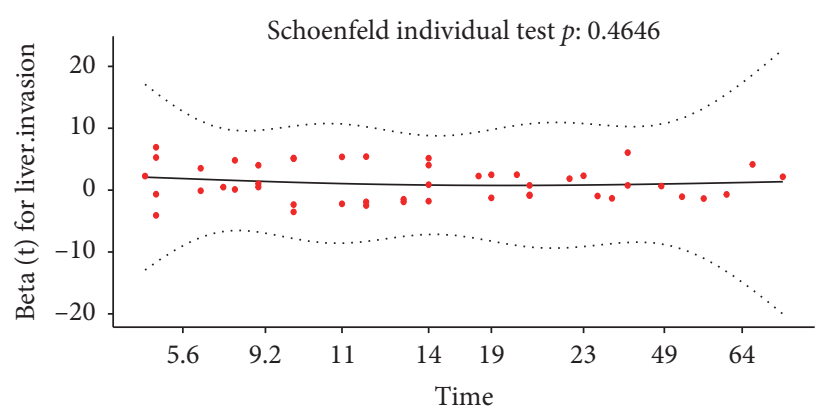

Global schoenfeld test $p: 0.3456$

(a)

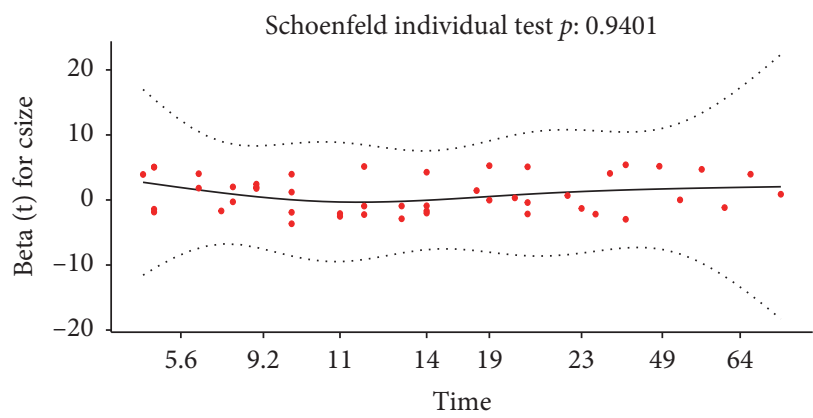

Global schoenfeld test $p: 0.3456$

(c)

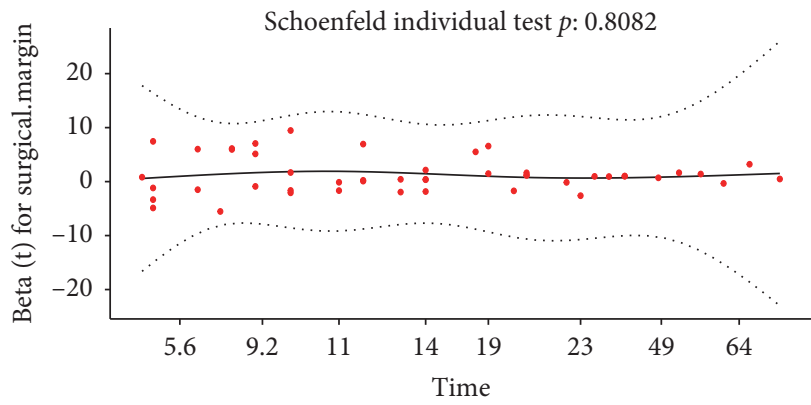

Global schoenfeld test p: 0.3456

(e)

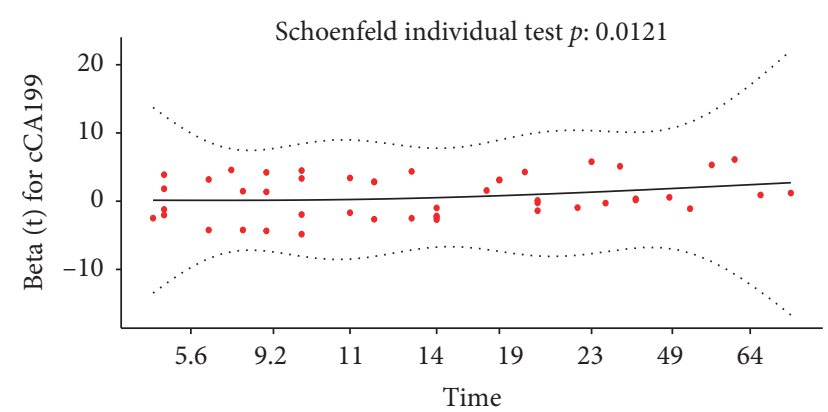

Global schoenfeld test $p: 0.3456$

(b)

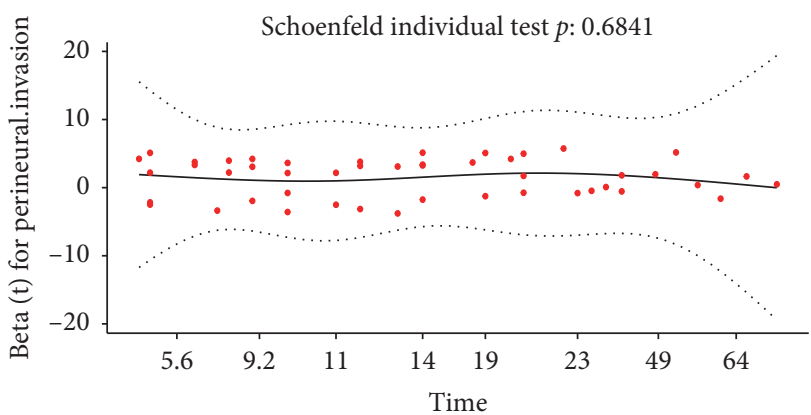

Global schoenfeld test $p: 0.3456$

(d)

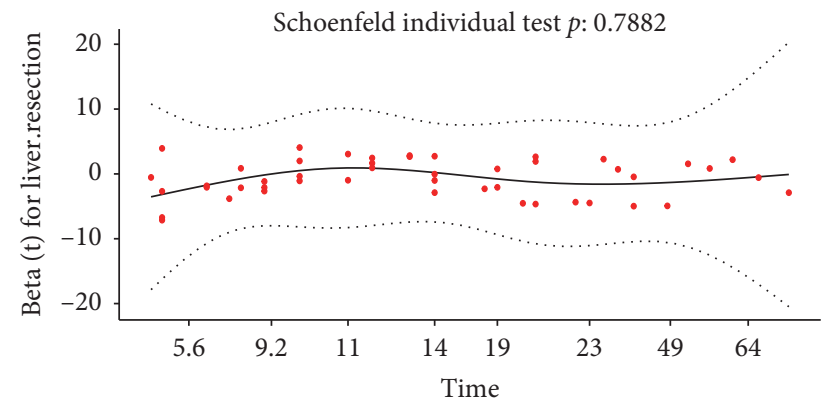

Global schoenfeld test $p: 0.3456$

(f)

FiguRE 1: Schoenfeld residuals analysis of liver invasion (a), cCA19-9 (b), ctumor size (c), perineural invasion (d), surgical margin (e), and liver resection (f).

the microenvironment of the host nerve [17]. Interestingly, many researches had indicated that tumor size may not be the independent risk factor for the prognosis of the GBC patients $[10,18]$. However, after transforming the tumor size into a categorical variable, we found that GBC patients with tumor sizes $<4.5 \mathrm{~cm}$ may have better prognoses.

A small and single-center retrospective study suggested that liver invasion may be the only independent risk factor of GBC even at a very early stage [19]. In our study, we confirmed that liver invasion affected significantly on the prognosis of GBC patients treated after surgery. Liver resection is an essential part of the radical surgery for the GBC patients at T1b or higher stage. There were two alternative hepatectomy choices for GBC, which were hepatic wedge resection around the gallbladder fossa or hepatic IVb/V segmentectomy. The purpose of the former operation was to obtain a negative resection margin, while the latter operation was anatomic resection to obtain an additional oncologic benefit [20]. Recently, a multicenter study showed that T2 GBC patients who underwent the liver resection had better five-year survival rate compared to those who received no liver resection [21]. Moreover, their results indicated patients who underwent hepatic wedge resection or IVb/V segmentectomy had similar disease-free survival. Other two multicenter studies also reported that there was no difference in survival rate or recurrence rates between the groups that underwent hepatic wedge resection and IVb/V segmentectomy $[22,23]$. In our center, most of GBC patients received the hepatic wedge resection and achieved negative resection margin. Large multicenter randomized control trials (RCTs) or matched studies regarding the safety and effectiveness of hepatic wedge resection versus IVb/V segmentectomy in GBC are needed in the future. 
Points

Liver.invasion

Csize

Perineural.invasion

Surgical.margin

Liver.resection

Total points

1-year survival probability

3-year survival probability

5-year survival probability

$\begin{array}{lllllllllll}0 & 10 & 20 & 30 & 40 & 50 & 60 & 70 & 80 & 90 & 100\end{array}$

Present

$$
\text { Absent }
$$

$<4.5$

Present

\section{Absent}

R1

$\mathrm{R} 0$

Present

Absent

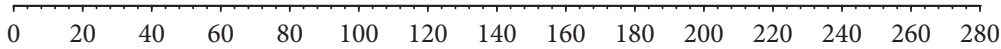

$\begin{array}{lllllllll}0.9 & 0.8 & 0.7 & 0.6 & 0.5 & 0.4 & 0.3 & 0.2 & 0.1\end{array}$

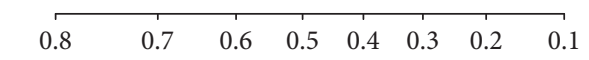

$\begin{array}{llllllll}0.8 & 0.7 & 0.6 & 0.5 & 0.4 & 0.3 & 0.2 & 0.1\end{array}$

FIgURE 2: The nomogram for predicting the 1-, 3-, and 5-year survival of gallbladder cancer patients.

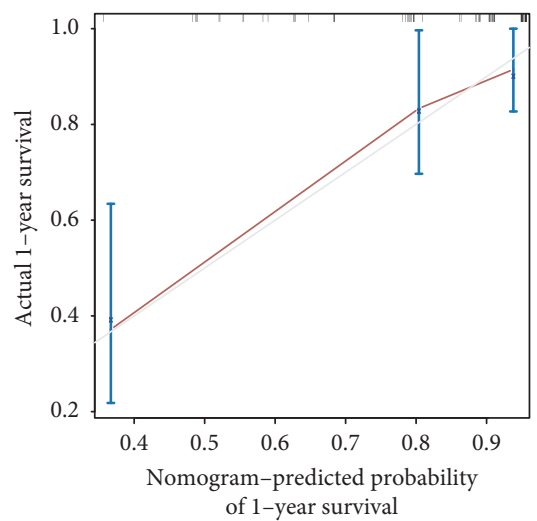

(a)

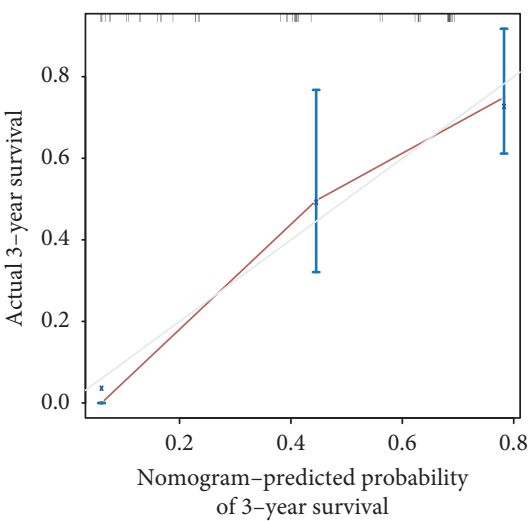

(b)

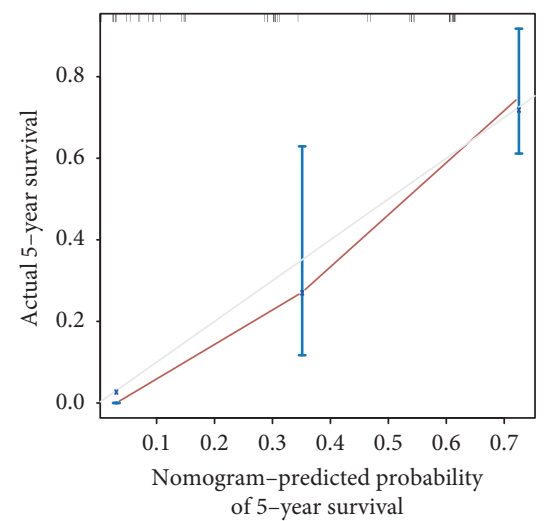

(c)

FigURE 3: Calibration plots of the nomogram for 1-year (a), 3-year (b), and 5-year (c) survival prediction of gallbladder cancer patients.

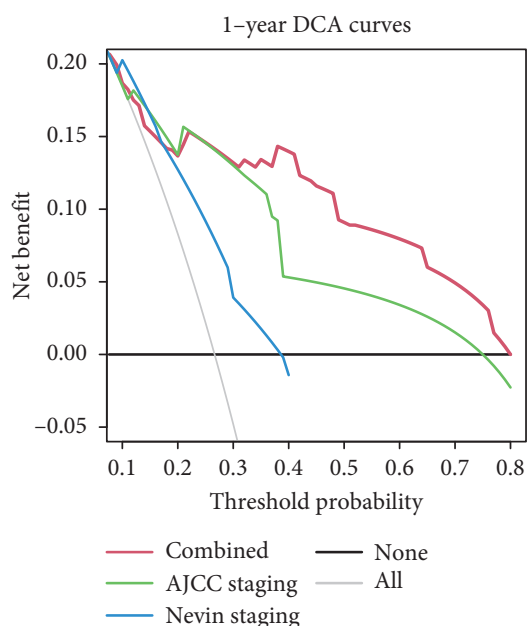

(a)

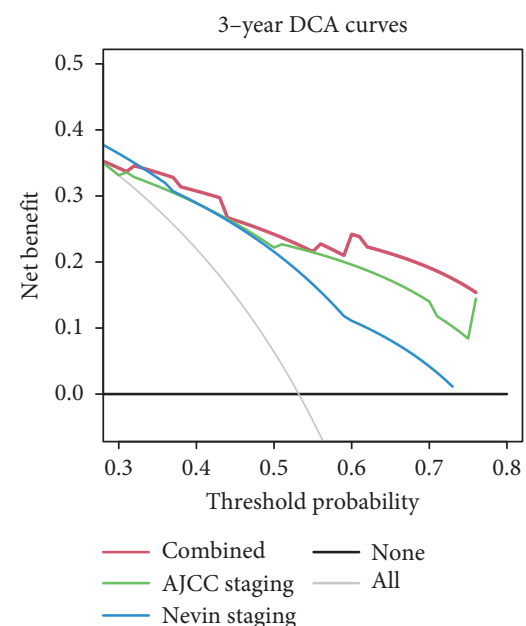

(b)

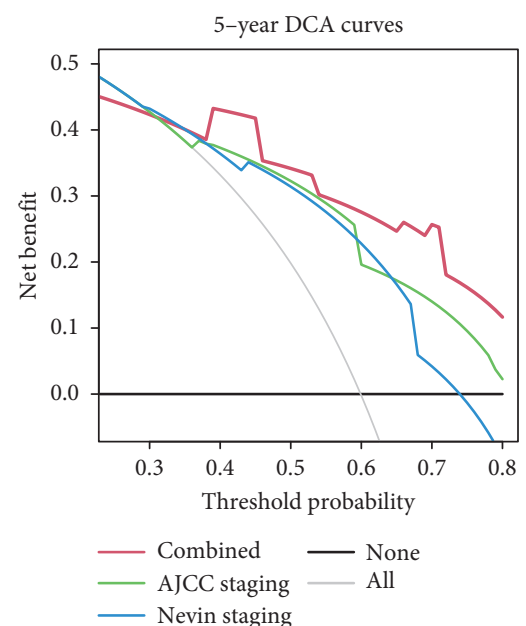

(c)

FIgURE 4: Decision curve analysis and Kaplan-Meier analysis of the nomogram. (A-C) Decision curve analysis of the nomogram for 1-year (a), 3-year (b), and 5-year (c) survival prediction of gallbladder cancer patients. 

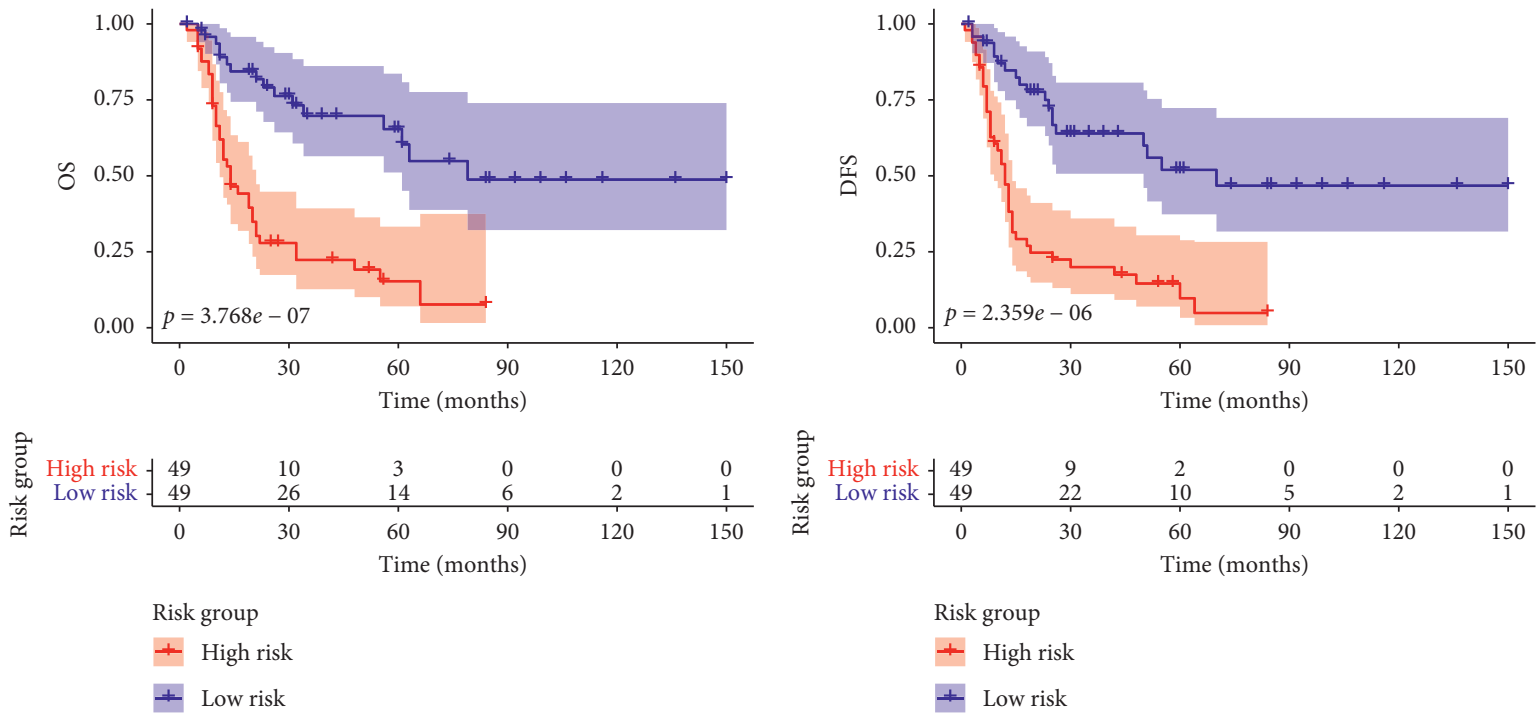

(a)

(b)

Figure 5: Kaplan-Meier analysis of gallbladder cancer patients stratified by median risk score. Patients in the high-risk group had a significantly shorter overall survival (a) and disease-free survival (b) than those in the low-risk group.

There are some limitations in our study. First, our nomogram may not be suitable for all GBC patients. Only patients without distant metastasis and who received surgical resection fit the model. Besides, the study was based on data from a single institute with a relatively small sample size. Independent cohorts from other centers are needed for future validation studies.

In conclusion, liver resection, tumor size, perineural invasion, surgical margin, and liver invasion played crucial roles in the prognosis of $\mathrm{GBC}$ patients. Based on these clinicopathological risk factors, we developed and validated a novel nomogram to predict the overall survival for GBC patients following surgical resection, which may facilitate decision-making in the clinic.

\section{Abbreviations}

GBC: Gallbladder cancer

TNM: Tumor-node-metastasis

AJCC: American Joint Committee on Cancer

SEER: Surveillance, Epidemiology, and End Results

CA19-9: Carbohydrate antigen 19-9

CA125: Carbohydrate antigen 125

CEA: Carcinoembryonic antigen

LNR: Lymph node positive rate

OS: Overall survival

DFS: Disease-free survival

ROC: Receiver operating characteristic curve

DCA: Decision curve analysis.

\section{Data Availability}

The underlying data supporting the results of our study are provided in the article.

\section{Disclosure}

Zuyi Ma, Fengying Dong, Zhenchong Li, and Zehao Zheng are co-first authors.

\section{Conflicts of Interest}

The authors declare that they have no potential conflicts of interest.

\section{Authors' Contributions}

Zuyi Ma, Yuanfeng Gong, Chuanzhao Zhang, and Baohua Hou conceived and designed the study. Fengying Dong, Zehao Zheng, and Zhenchong Li identified and included eligible subjects. Hongkai Zhuang, Chunsheng Liu, Bowen Huang, Yiping Zou, Shanzhou Huang, and LinLing Yang extracted data from electronic medical records. Zuyi Ma, Fengying Dong, Zehao Zheng, and Zhenchong Li carried out the statistical analysis and interpreted the results. All drafts of the reports were written by Zuyi Ma and Baohua Hou. All authors read and approved the final version of the manuscript. Zuyi Ma, Fengying Dong, Zhenchong $\mathrm{Li}$, and Zehao Zheng contributed equally to this work.

\section{Acknowledgments}

The authors would like to thank everyone who participated in this study. This study was supported by Special Funding for Ph.D. Doctor of Guangdong Provincial People's Hospital (project no.: 2020bq09), National Natural Science Foundation of China (project nos.: 82072635, 81702783, 82072637, and 81672475), and Guangdong Medical Science and Technology Fund (project no.: 201707010323). 


\section{References}

[1] F. Bray, J. Ferlay, I. Soerjomataram, R. L. Siegel, L. A. Torre, and A. Jemal, "Global cancer statistics 2018: GLOBOCAN estimates of incidence and mortality worldwide for 36 cancers in 185 countries," CA: A Cancer Journal for Clinicians, vol. 68, no. 6, pp. 394-424, 2018.

[2] M. Rakić, L. Patrlj, M Kopljar et al., "Gallbladder cancer," Hepatobiliary Surgery and Nutrition, vol. 3, no. 5, pp. 221-226, 2014.

[3] Z. Wen, A. Si, J. Yang et al., "Elevation of CA19-9 and CEA is associated with a poor prognosis in patients with resectable gallbladder carcinoma," $H p b$, vol. 19, no. 11, pp. 951-956, 2017.

[4] S. Yamashita, G. Passot, T. A. Aloia et al., "Prognostic value of carbohydrate antigen 19-9 in patients undergoing resection of biliary tract cancer," British Journal of Surgery, vol. 104, no. 3, pp. 267-277, 2017.

[5] E. A. Vega, E. Vinuela, M. Sanhueza et al., "Positive cystic duct margin at index cholecystectomy in incidental gallbladder cancer is an important negative prognosticator," European Journal of Surgical Oncology, vol. 45, no. 6, pp. 1061-1068, 2019.

[6] G. A. Margonis, F. Gani, S. Buettner et al., "Rates and patterns of recurrence after curative intent resection for gallbladder cancer: a multi-institution analysis from the US Extra-hepatic Biliary Malignancy Consortium," $H p b$, vol. 18, no. 11, pp. 872-878, 2016.

[7] R. Higuchi, T. Yazawa, S Uemura et al., "Examination of prognostic factors affecting long-term survival of patients with stage $3 / 4$ gallbladder cancer without distant metastasis," Cancers (Basel), vol. 12, 2020.

[8] J. E. Nevin, T. J. Moran, S. Kay, and R. King, "Carcinoma of the gallbladder. Staging, treatment, and prognosis," Cancer, vol. 37, no. 1, pp. 141-148, 1976.

[9] M. B. Amin, F. L. Greene, S. B. Edge et al., "The Eighth Edition AJCC Cancer Staging Manual: continuing to build a bridge from a population-based to a more "personalized" approach to cancer staging," CA: A Cancer Journal for Clinicians, vol. 67, no. 2, pp. 93-99, 2017.

[10] M. Chen, J. Cao, B. Zhang, L. Pan, and X. Cai, "A nomogram for prediction of overall survival in patients with node-negative gallbladder cancer," Journal of Cancer, vol. 10, no. 14, pp. 3246-3252, 2019.

[11] S. J. Wang, A. Lemieux, J. Kalpathy-Cramer et al., "Nomogram for predicting the benefit of adjuvant chemoradiotherapy for resected gallbladder cancer," Journal of Clinical Oncology, vol. 29, no. 35, pp. 4627-4632, 2011.

[12] T. A. Aloia, N. Járufe, M. Javle et al., "Gallbladder cancer: expert consensus statement," $H p b$, vol. 17, no. 8, pp. 681-690, 2015.

[13] B.-W. Kim, C.-M. Oh, H. Y. Choi, J.-W. Park, H. Cho, and $\mathrm{M}$. $\mathrm{Ki}$, "Incidence and overall survival of biliary tract cancers in South Korea from 2006 to 2015: using the national health information database," Gut and Liver, vol. 13, no. 1, pp. 104-113, 2019.

[14] H. Kijima, Y. Wu, T. Yosizawa et al., "Pathological characteristics of early to advanced gallbladder carcinoma and extrahepatic cholangiocarcinoma," Journal of Hepato-BiliaryPancreatic Sciences, vol. 21, no. 7, pp. 453-458, 2014.

[15] S. Maruyama, H. Kawaida, N Hosomura et al., "Indications for extrahepatic bile duct resection due to perineural invasion in patients with gallbladder cancer," World Journal of Surgical Oncology, vol. 17, 2019.
[16] H. Lee, D. W. Choi, J. Y. Park et al., "Surgical strategy for T2 gallbladder cancer according to tumor location," Annals of Surgical Oncology, vol. 22, no. 8, pp. 2779-2786, 2015.

[17] R. Bakst and R. Wong, "Mechanisms of perineural invasion," Journal of Neurological Surgery Part B: Skull Base, vol. 77, no. 02, pp. 096-106, 2016.

[18] Y. Wu, Q. Li, Z. Cai et al., "Survival prediction for gallbladder carcinoma after curative resection: comparison of nomogram and Bayesian network models," European Journal of Surgical Oncology, vol. 46, no. 11, pp. 2106-2113, 2020.

[19] M. D’Hondt, R. Lapointe, Z. Benamira et al., "Carcinoma of the gallbladder: patterns of presentation, prognostic factors and survival rate. An 11-year single centre experience," European Journal of Surgical Oncology, vol. 39, pp. 548-553, 2013.

[20] S. K. Reddy and B. M. Clary, "Surgical management of gallbladder cancer," Surgical Oncology Clinics of North America, vol. 18, no. 2, pp. 307-324, 2009.

[21] W. Kwon, H. Kim, Y Han et al., "Role of tumour location and surgical extent on prognosis in T2 gallbladder cancer: an international multicentre study," British Journal of Surgery, vol. 107, no. 10, pp. 1334-1343, 2020.

[22] A. Horiguchi, S. Miyakawa, S. Ishihara et al., "Gallbladder bed resection or hepatectomy of segments $4 \mathrm{a}$ and 5 for pT2 gallbladder carcinoma: analysis of Japanese registration cases by the study group for biliary surgery of the Japanese society of hepato-biliary-pancreatic surgery," Journal of Hepato-Biliary-Pancreatic Sciences, vol. 20, no. 5, pp. 518-524, 2013.

[23] T. Araida, R. Higuchi, M. Hamano et al., "Hepatic resection in 485 R0 pT2 and pT3 cases of advanced carcinoma of the gallbladder: results of a Japanese Society of Biliary Surgery survey-a multicenter study," Journal of Hepato-Biliary-Pancreatic Surgery, vol. 16, no. 2, pp. 204-215, 2009. 